\title{
Small and large scale site diversity experiment at Ka-band in the south of France
}

\author{
Xavier Boulanger ${ }^{1, *, \dagger}$, Frédéric Lacoste ${ }^{2}$ and Laurent Castanet $^{1}$ \\ ${ }^{1}$ ONERA - Université Fédérale de Toulouse (UFTMiP), ElectroMagnetics \& Radar Department, RadioCommunications and \\ Propagation research unit, 2 Avenue Edouard Belin, BP 74025, 31055 Toulouse Cedex 4, France \\ ${ }^{2}$ Centre National d'Etudes Spatiales (CNES), DCT/RF/ITP, 18 Avenue Edouard Belin, 31401 Toulouse Cedex 4, France
}

\begin{abstract}
SUMMARY
Since April 2011, ONERA has been operating in Toulouse, France, a beacon receiver able to receive the $20.199 \mathrm{GHz}$ beacon signal of ASTRA 3B. From June 2013 to December 2013, ONERA and CNES successively deployed in the South of France four more beacon receivers in order to characterize the space-time behaviour of the propagation channel at Ka-band. This Site Diversity configuration is of great interest for the development of future high data rate satellite services as it efficiently mitigates severe tropospheric propagation impairments, in particular rain attenuation which is the major issue. The ONERA-CNES Ka-band site diversity experiment consists of three beacon receivers deployed in the area of Toulouse with site separation between 16 and $26 \mathrm{~km}$, and two additional beacon receivers at $140 \mathrm{~km}$ and $300 \mathrm{~km}$ from Toulouse. This paper aims at providing a complete description of the experimental set-up and the statistical results of the campaign from July 2013 to December 2014. The Complementary Cumulative Distribution Functions (CCDF) of rain attenuation on each site and the Joint Distributions for several pairs of radio links are illustrated. Some comparisons of the measured Diversity Gain with two state-of-the-art prediction models are also presented.
\end{abstract}

KEY WORDS: diversity gain; propagation; rain attenuation; satellite communications; site diversity

\section{INTRODUCTION}

More and more satellite communication systems are being deployed at high frequency bands, especially at Ka-band, to get higher bandwidth in order to offer high data rate multimedia services. Ka-Sat [1] in Europe, ViaSat-1 in the USA, and Yahsat in the Middle East [2] are recent examples of such high throughput systems. Future Satcom systems with feeder links operating at Q/V-band [3] are currently studied to benefit from larger bandwidth and to reach Tb/s capacity [4]. However, the use of such high frequency bands (Ka- and Q/V-bands) is limited by the influence of the propagation channel through the troposphere whose impact can be reduced by the use of Fade Mitigation Techniques (FMT) [5]. The implementation of gateways in Site Diversity configuration appears as a very efficient mitigation technique to counteract severe propagation impairments on the feeder links [5-7], in particular rain attenuation which is the major issue. Indeed, Site Diversity consists in separating the ground terminals by distances ranging from several to tens of kilometres to benefit from the spatial decorrelation of the rain cells. As the most intense rain cells, i.e. those producing the severest attenuation, are generally quite limited in horizontal extent [8], the probability of simultaneous rain attenuation occurring on several network routes is less than the probability of rain attenuation occurring on each individual slant path. At $\mathrm{Q} / \mathrm{V}$ bands, it is probably not always realistic to consider

*Correspondence to: Xavier Boulanger, ONERA - Université Fédérale de Toulouse (UFTMiP), ElectroMagnetics \& Radar Department, RadioCommunications and Propagation research unit, 2 Avenue Edouard Belin, BP 74025, 31055 Toulouse Cedex 4, France ${ }^{\dagger}$ E-mail: xavier.boulanger@onera.fr 
the duplication of gateways due to prohibitive cost of deployment. Nevertheless, the concept of "smart gateways" has been introduced in [3] where the performances of different macro-diversity schemes have been assessed. It allows the deep fades experienced at Q/V bands to be mitigated by introducing cooperation and a limited redundancy between the different gateways of the system. Even for large site separation distances, the design of such systems may require investigations of the space-time propagation channel.

The influence of the propagation channel for the Fixed Satellite Service has been extensively studied in the past thirty years and propagation prediction methods have been developed and tested with respect to experimental campaigns [5]. However, the major part of these propagation experiments only addressed single-site configurations (mainly in Europe [9-16], in Japan [17, 18] and North America [16] and [19-21]). Quite few beacon measurements were carried out in site diversity configuration [22-25]. If most of them investigated large-scale schemes [22, 23], few addressed specific small-scale site separations [24, 25]. Moreover, the experiments durations were generally too short to provide reliable long-term statistics. For instance, in France, only one experiment was carried out in the 1990s by France Telecom R\&D with two Earth Stations separated by a distance of $5 \mathrm{~km}$ and over a period of 6 months [9].

Recently, in order to characterize the space-time behaviour of the propagation channel at Ka-band, CNES and ONERA have decided to carry out a new Site Diversity propagation experiment to collect propagation data at Ka-band from a network of five beacon receivers deployed in the South of France. Three of them had to cover a small scale diversity configuration in the area of Toulouse. The experiment was based on the acquisition of the $20.199 \mathrm{GHz}$ beacon signal (vertical polarization) of the satellite ASTRA 3B.

The aim of this paper is to present the statistical results of the propagation campaign from July 2013 to December 2014. In Section 2, the ONERA-CNES Ka-band Site Diversity experiment is presented. First, a general overview of the ground segment and link parameters is given. Secondly, the experimental set-up is described. Section 3 provides the statistical results of the experiment. The single-site Complementary Cumulative Distribution Functions (CCDF) of rain attenuation and the Joint Distributions for several pairs of radio links are illustrated. Finally, some comparisons of the measured Diversity Gain with prediction models of Rec. ITU-R P.618-12 [26] are proposed in Section 4.

\section{THE ONERA-CNES KA-BAND SITE DIVERSITY EXPERIMENT}

\subsection{Overview of the ground segment}

From January 2008 to March 2011, ONERA operated a beacon receiver able to acquire the $19.7 \mathrm{GHz}$ beacon signal of the EUTELSAT HotBird 6 satellite [27]. In March 2011, the RF chain was modified to be able to receive the $20.199 \mathrm{GHz}$ ASTRA 3B beacon (located on a geostationary orbit at $23.5^{\circ}$ of longitude) to benefit from a higher EIRP of the satellite [27]. It allows a higher dynamic range of the receiver to be reached ( $\sim 37 \mathrm{~dB}$ with ASTRA 3B instead of $\sim 26 \mathrm{~dB}$ with HotBird 6) [27].

From July 2013 to December 2013, ONERA and CNES equipped four more ASTRA 3B measurement sites. Figure 1 shows the general overview of the ONERA-CNES Ka-band site diversity experiment. Large-scale site diversity is established between Toulouse and Aire sur l'Adour $(\sim 140 \mathrm{~km})$ and between Toulouse and Salon de Provence $(\sim 300 \mathrm{~km})$. Small-scale site diversity is carried out between Toulouse, Aussaguel and Le Fauga (16-26 km). It is worth noting that Aire sur l'Adour and the three sites around Toulouse are more or less aligned with the dominant wind flux direction. Table I provides the locations of the sites and the radio link parameters. Table II gives the distances between each site.

\subsection{Description of the experimental set-up}

2.2.1. Measurement site in Toulouse. The experimental set-up (still operational) in Toulouse is the following [27]:

- an indoor beacon receiver designed by ONERA,

- a profiling Radiometrics TP-WVP 3000 radiometer (ONERA), 


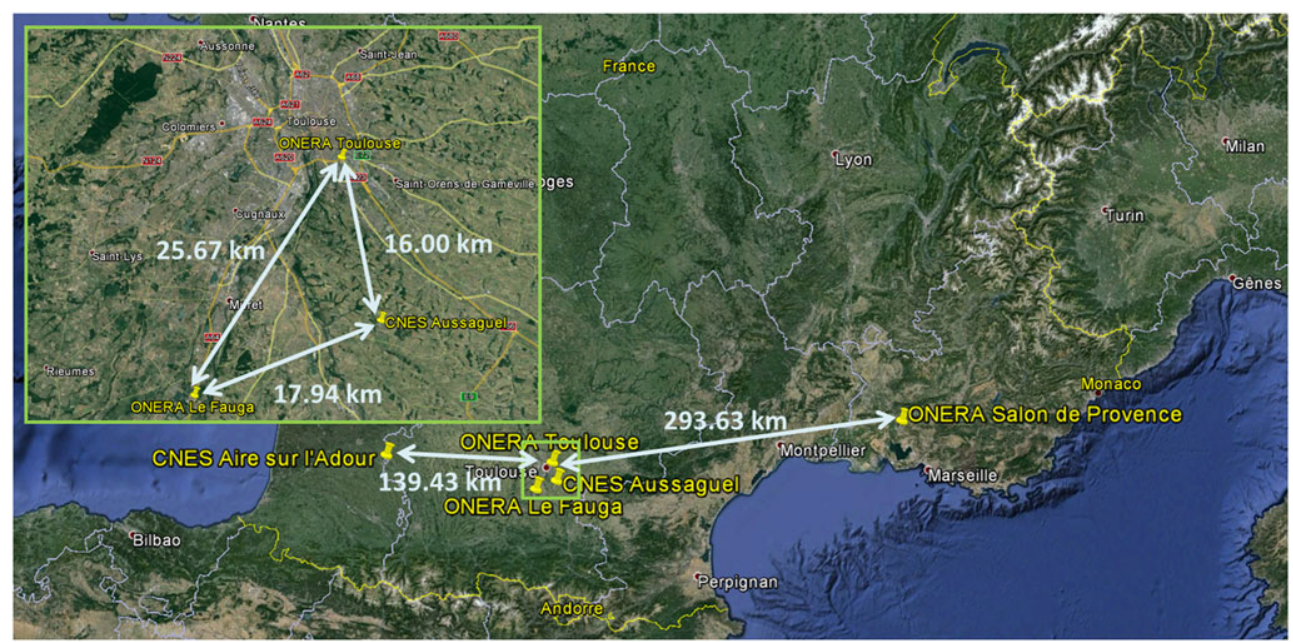

Figure 1. Overview of the ONERA-CNES Ka-band site diversity experiment.

Table I. Locations of the sites and link parameters of the ONERA-CNES Ka-band site diversity experiment.

\begin{tabular}{lccccc}
\hline Location & Latitude $\left({ }^{\circ} \mathrm{N}\right)$ & Longitude $\left({ }^{\circ} \mathrm{E}\right)$ & Altitude $(\mathrm{km})$ & Elevation $\left({ }^{\circ}\right)$ & Azimuth $\left({ }^{\circ}\right)$ \\
\hline Toulouse & 43.5710 & 1.4716 & 0.150 & 35.11 & 149.57 \\
Aussaguel & 43.4284 & 1.4982 & 0.195 & 35.26 & 149.53 \\
Le Fauga & 43.3842 & 1.2846 & 0.190 & 35.21 & 149.25 \\
Aire sur l'Adour & 43.7061 & -0.2510 & 0.081 & 34.27 & 147.49 \\
Salon de Provence & 43.6192 & 5.1175 & 0.067 & 36.43 & 154.26 \\
\hline
\end{tabular}

Table II. Distances between each site of the ONERA-CNES Ka-band site diversity experiment.

\begin{tabular}{lccccc}
\hline Distances $(\mathrm{km})$ & Toulouse & Aussaguel & Le Fauga & Aire sur l'Adour & Salon de Provence \\
\hline Toulouse & 0 & 16.00 & 25.67 & 139.43 & 293.63 \\
Aussaguel & & 0 & 17.94 & 144.27 & 292.56 \\
Le Fauga & & 0 & 128.84 & 310.22 \\
Aire sur l'Adour & & & 0 & 431.88 \\
Salon de Provence & & & & 0 \\
\hline
\end{tabular}

- a tipping bucket rain gauge (ONERA).

Basically, the beacon receiver consists of a Cassegrain parabolic antenna of $1.20 \mathrm{~m}$ diameter with the RF chain immediately placed at the output of the antenna to avoid feeder loss. Three frequency down-conversions are performed: a first one from $20.199 \mathrm{GHz}$ to $110 \mathrm{MHz}$, a second one from $110 \mathrm{MHz}$ to $10 \mathrm{MHz}$ and a third one from $10 \mathrm{MHz}$ to $2 \mathrm{kHz}$. The dynamic range is around $37 \mathrm{~dB}$ using the ASTRA 3B beacon signal. For a more detailed description of the equipment, the reader may refer to [27].

2.2.2. Measurement site in Aussaguel. The equipment deployed in Aussaguel were the following (Figure 2):

- an outdoor beacon receiver (CNES),

- a dual-channel RPG LWP radiometer (CNES).

The CNES outdoor beacon receiver antenna is a $1.2 \mathrm{~m}$ offset parabolic antenna. The signal picked up by the antenna undergoes a first low noise frequency conversion from $20.199 \mathrm{GHz}$ to $105 \mathrm{MHz}$ performed by the LNBC (Low Noise Block-Converter). These blocks are made up of a very-low-noise RF 


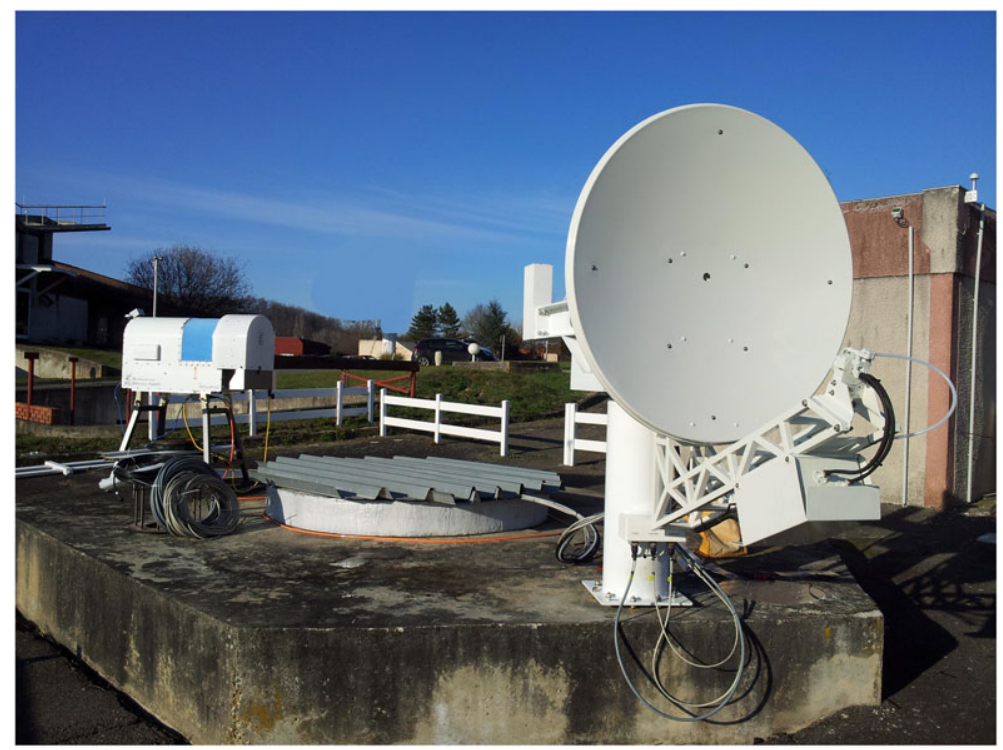

Figure 2. CNES outdoor beacon receiver and dual channel RPG LWP radiometer installed at CNES Aussaguel premises.

amplifier, a guiding filter for rejection of image frequency, a millimetric mixer and an IF amplifier. The mixer local oscillator is provided by a double loop PLDRO (Phase-Locked Dielectric Resonator Oscillator) referenced by an internal OCXO (Oven Controlled X-tal (Crystal) Oscillator). Both the LNBC and the PLDRO are incorporated in a watertight unit near the antenna source. A temperature sensor is incorporated in the unit to measure the temperature for possible thermal drift compensation of the chain. The $105 \mathrm{MHz}$ useful signal is then sent to the receiver rack through a low-loss double-braided coaxial cable, with a maximum $20 \mathrm{~m}$ length. Then, the signal undergoes a second frequency conversion from 105 to $5 \mathrm{MHz}$. This second conversion aims at eliminating interferences, at reducing the noise band and at providing a signal with a frequency suited to the DSP board acquisition chain. Finally, the signal is sampled and processed by a DDS card. Once acquired, a frequency search and a frequency tracking of the test signal is performed to be able to integrate the CW power over every small frequency bandwidth (typically $50 \mathrm{~Hz}$ ). The receive power is computed by simply integrating the signal Power Spectral Density over this measurement bandwidth. The equipment is composed of two different modules:

- the outdoor module: antenna, RF front end (up to the first frequency conversion), GPS antenna (for time reference), rain sensor (to detect rainy conditions),

- the indoor module: DSP board, dry air compressor (to ensure stable humidity and pressure conditions of the RF front end), air compressor (to blow air on the feed radome when rain is detected), MMI computer, and antenna motion controller.

This equipment was originally dedicated to an experiment over India. Consequently, its design takes into account tropical constraints (high wind speed, humidity, rain intensity, and high elevation angle). With this receiver, the dynamic range is around $37 \mathrm{~dB}$ using the ASTRA 3B beacon signal.

\subsubsection{Measurement site in Le Fauga. The equipment deployed in Le Fauga were the following:}

- an outdoor beacon receiver (CNES),

- a profiling RPG HATPRO radiometer (ONERA),

- a tipping bucket rain gauge (ONERA).

The CNES outdoor beacon receiver deployed in Le Fauga was similar to the one described in Section 2.2.2 (as for the signal processing).

2.2.4. Measurement site in Aire sur l'Adour. The beacon receiver deployed in Aire sur l'Adour is a "simplified version" of the outdoor equipment presented in Section 2.2.2. It was also designed by 
Assystem France, to be operated in typical European conditions. Consequently, environmental requirements were less critical. In particular, no air is blown on the RF front end block or on the feed radome. The antenna pointing system is manual. To make it more generic, a commercial thermally stabilized Ka-band to L-band LNBC (from Orbital Science, Canada) is used and then, a software-controlled Lband to $5 \mathrm{MHz}$ board was specifically designed. As a matter of fact, it can be used for a large bandwidth at L-band which makes it easily reusable for different experiments between $19.7 \mathrm{GHz}$ and 20.2 GHz. Then, the signal processing is exactly the same than for the CNES outdoor beacon receivers and already described in Section 2.2.2.

Since the outdoor module is pretty much lighter than for the CNES beacon receivers, it can be more easily accommodated over a rooftop (see Aire sur l'Adour and Salon de Provence configurations). The simplified beacon receiver installed in Aire sur l'Adour was the prototype version, originally designed with a $0.9 \mathrm{~m}$ Patriot offset parabolic antenna (see left part of Figure 3).

2.2.5. Measurement site in Salon de Provence. The equipment deployed in Salon de Provence were the following:

- a simplified beacon receiver (ONERA),

- a profiling RPG HATPRO radiometer (CNES),

- a tipping bucket rain gauge (ONERA).

The beacon receiver was a recurrent version of the one described in Section 2.2.4. Validation tests performed on the prototype simplified beacon receiver showed that the original design was not optimal because of possible accumulation of water droplets on the feed. For the second device to be deployed in Salon de Provence, it has been decided to switch to a $0.9 \mathrm{~m}$ prime focus parabolic antenna (see right part of Figure 3).

\subsection{Data processing and availability}

To get high quality rain attenuation time series, the data processing methodology described in [27] was used. When concurrent radiometric measurement data were available, they have been used to improve accuracy in the identification of rainy periods but also to generate, when possible, time series of total attenuation. First, the methodology consists in identifying periods for which integrated liquid water content is over $1 \mathrm{~mm}$ (obtained by linear regression methods on attenuation given by the radiometer). These periods are then manually adjusted to avoid any discontinuity into the final attenuation time

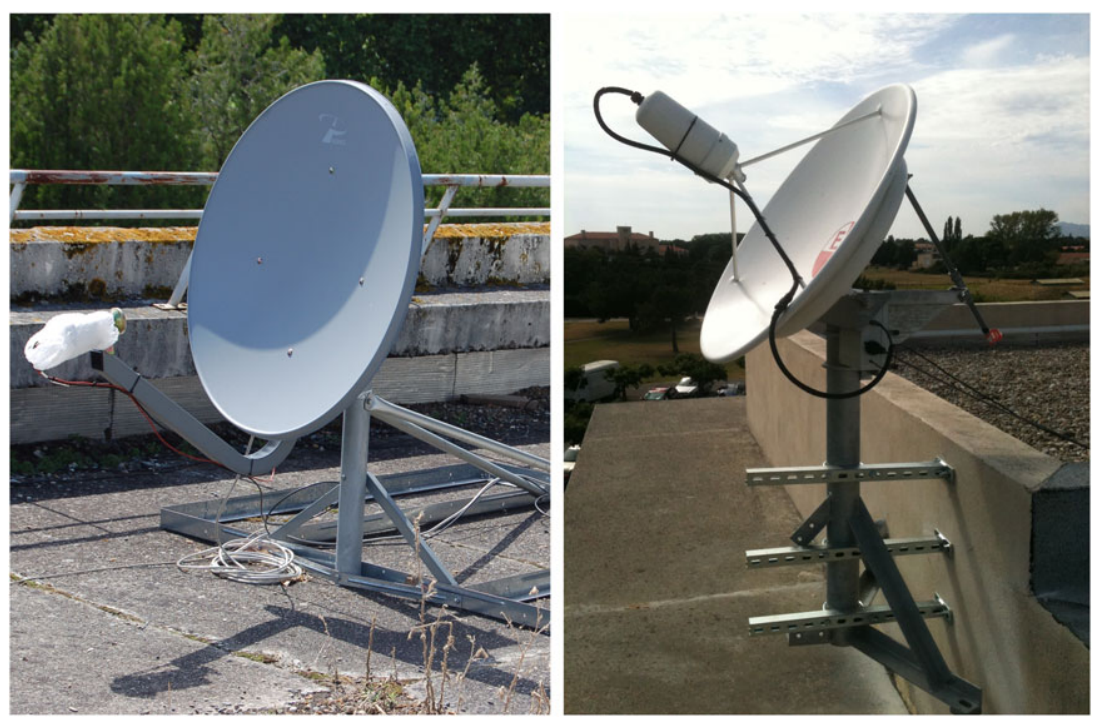

Figure 3. Simplified beacon receiver (left: prototype version deployed at Aire sur l'Adour premises, right: $2^{\text {nd }}$ version deployed at Salon de Provence premises). 
series. When radiometric data are not available, an FFT filtering of the raw data is only performed to get the equivalent $0 \mathrm{~dB}$ reference level.

The periods and the availabilities of measurements for each site are highlighted in Table III. The availability is defined as the total valid samples (identified after data processing) divided by the total observation period. As an example, Figure 4 shows concurrent processed time series of rain attenuation recorded at $20.2 \mathrm{GHz}$ using ASTRA 3B beacon, from 3 to 4 April 2014, and for all the sites of the propagation experiment.

Table III. Periods and availabilities of measurements for each site.

\begin{tabular}{llc}
\hline Location & \multicolumn{1}{c}{ Period of measurements } & Availability of valid data (\%) \\
\hline Toulouse & July 2011 - December 2014 & 98.91 \\
Aussaguel & July 2013 - November 2014 & 92.95 \\
& (No data from December 2013 & \\
Le Fauga & to February 2014 and in June 2014) & 89.04 \\
Aire sur l'Adour & December 2013 - December 2014 & 96.48 \\
Salon de Provence & January 2014 - July 2014 & 92.79 \\
\hline
\end{tabular}
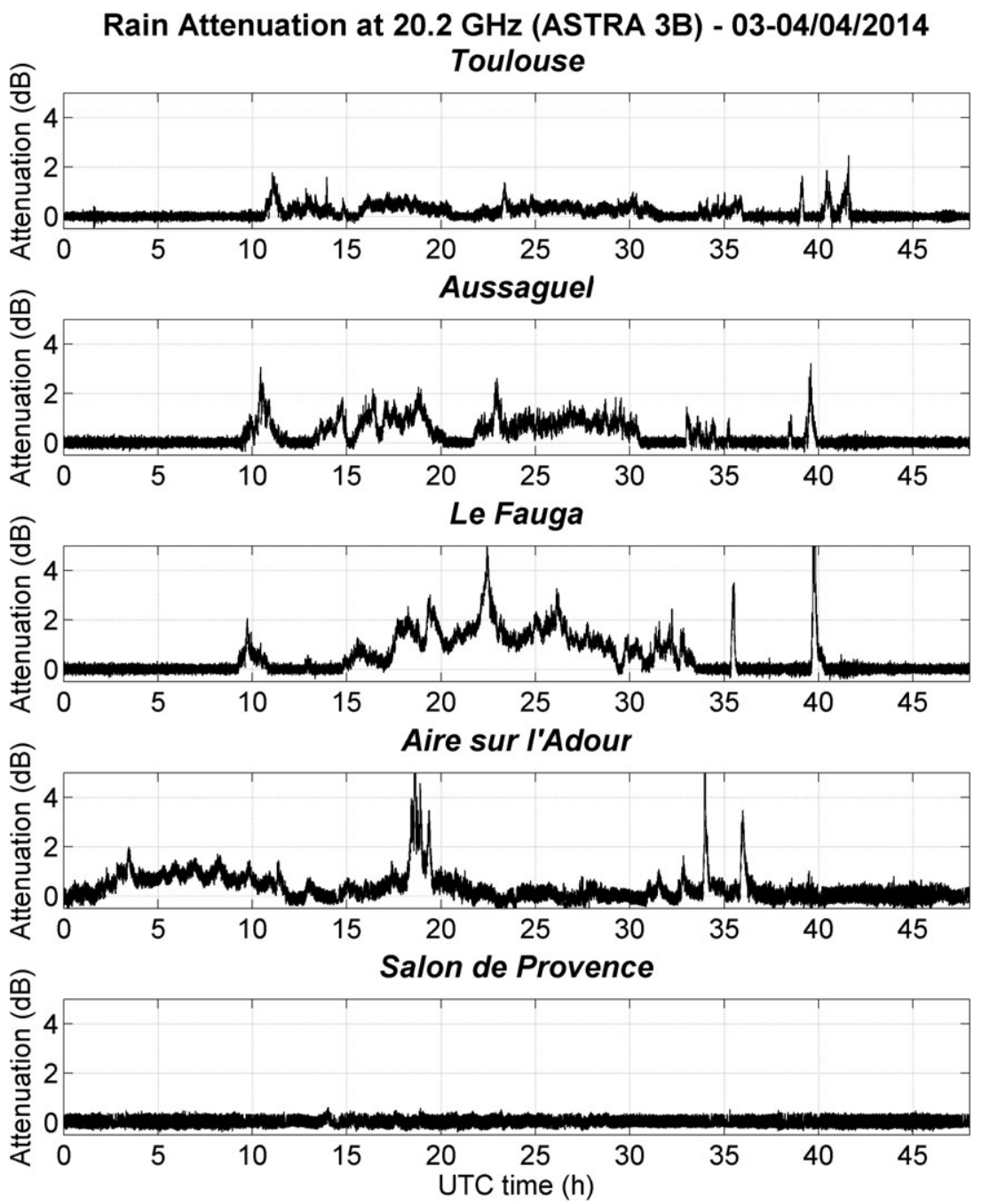

Figure 4. Example of concurrent processed time series of rain attenuation recorded at $20.2 \mathrm{GHz}$ using ASTRA 3B beacon from 3 to 4 April 2014. 


\section{STATISTICAL RESULTS}

\subsection{Small-scale site diversity configuration}

To analyse the small-scale site diversity results, the three measurement sites located in the area of Toulouse are considered, i.e. Toulouse, Le Fauga, and Aussaguel. For each pair of radio links, the single-site CCDF of rain attenuation and the experimental joint CCDF are computed. Moreover, to better illustrate the effect of the spatial correlation, the theoretical joint CCDF for the statistical independence case is also shown. This theoretical distribution can be easily obtained by multiplying the two single probabilities of exceedance for a given rain attenuation threshold. Finally, the correlation coefficient $r_{A_{i j}}$ of the unconditioned processes (i.e. taking into account rainy and non-rainy periods) between ground station $i$ and ground station $\mathrm{j}$ is computed. It is defined as:

$$
r_{A_{i j}}=\frac{E\left[A_{i}(t) A_{j}(t)\right]-E\left[A_{i}(t)\right] E\left[A_{j}(t)\right]}{\sqrt{E\left[A_{i}^{2}(t)\right]-E\left[A_{i}(t)\right]^{2}} \sqrt{E\left[A_{j}^{2}(t)\right]-E\left[A_{j}(t)\right]^{2}}}
$$

where $A_{i}(\mathrm{t})$ and $A_{j}(\mathrm{t})$ are the rain attenuation time series respectively on ground station $i$ and ground station $j$, and $\mathrm{E}[]$ is the expectation operator.

Figure 5 shows the CCDF of rain attenuation at $20.2 \mathrm{GHz}$ in site diversity configuration between Toulouse and Le Fauga. The statistics have been computed from December 2013 to December 2014 over $88.26 \%$ of concurrent valid data. The correlation coefficient is equal to 0.2281 .

Figure 6 shows the CCDF of rain attenuation at $20.2 \mathrm{GHz}$ in site diversity configuration between Toulouse and Aussaguel. The statistics have been computed over 13 months between July 2013 and November 2014 (as previously mentioned in Table III, there is no data from December 2013 to February 2014 and in June 2014 for Aussaguel measurement site). The availability of concurrent valid data is equal to $92.94 \%$. The correlation coefficient is equal to 0.3780 .

Figure 7 shows the CCDF of rain attenuation at $20.2 \mathrm{GHz}$ in site diversity configuration between Le Fauga and Aussaguel. The statistics have been computed over 8 months included between March 2014 and November 2014 (as previously mentioned in Table III, there is no data in June 2014 for Aussaguel measurement site). The availability of concurrent valid data is equal to $90.05 \%$. The correlation coefficient is equal to 0.2576 .

As a general trend, it can be highlighted from Figures 5-7 that the concurrent single-site CCDF of rain attenuation present similar shapes. Nevertheless, the concurrent distributions are not strictly the same showing the impact of the site separation even at small scale. We could also have expected closer correlation values between Toulouse/Aussaguel and Le Fauga/Aussaguel configurations for which the separation distances are almost identical. The baseline orientation might have an influence. Finally, it is

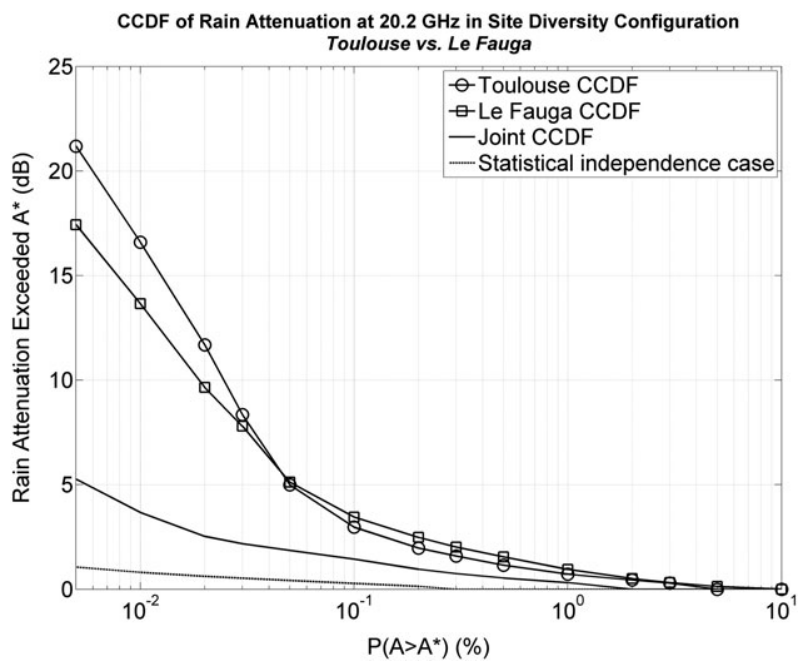

Figure 5. CCDF of rain attenuation at $20.2 \mathrm{GHz}$ in site diversity configuration between Toulouse and Le Fauga. 


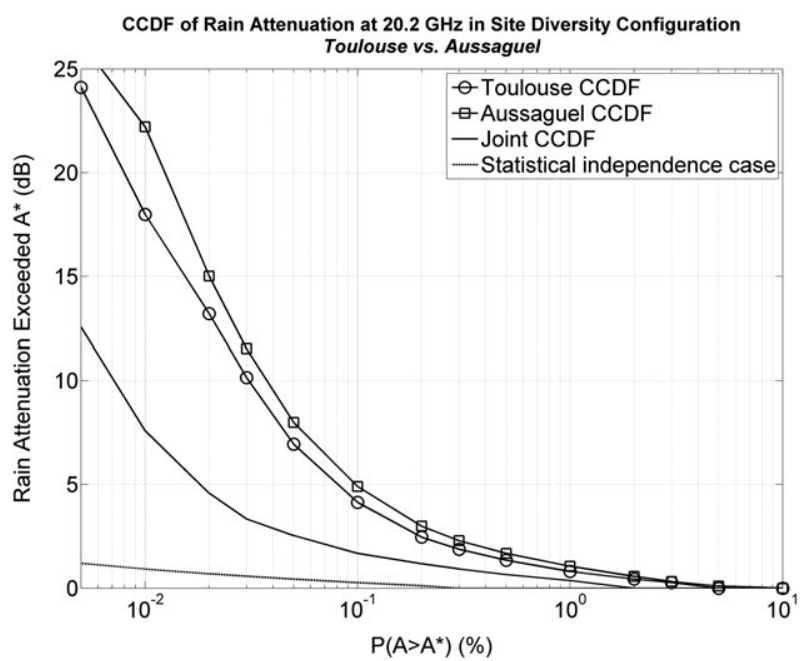

Figure 6. CCDF of rain attenuation at $20.2 \mathrm{GHz}$ in site diversity configuration between Toulouse and Aussaguel.

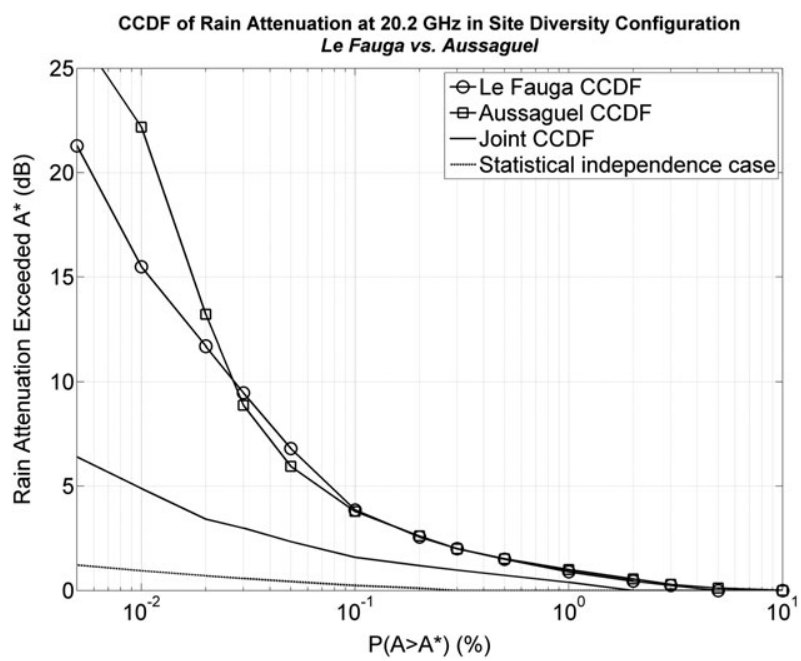

Figure 7. CCDF of rain attenuation at $20.2 \mathrm{GHz}$ in site diversity configuration between Le Fauga and Aussaguel.

not surprising to observe that the correlation coefficient strongly decreases with the distance between the measurement sites.

\subsection{Large-scale site diversity configuration}

For sake of simplicity, large-scale site diversity results are only illustrated in Toulouse/Salon de Provence and Toulouse/Aire sur l'Adour configurations.

Figure 8 shows the CCDF of rain attenuation at $20.2 \mathrm{GHz}$ in site diversity configuration between Toulouse and Salon de Provence. The statistics have been computed from July 2013 to December 2014 over $92.23 \%$ of concurrent valid data. The correlation coefficient is equal to 0.0273 . Contrary to the results presented from Figures 5-8 highlights a strong difference of the concurrent single-site CCDF. It has to be recalled that Toulouse and Salon de Provence are separated by almost $300 \mathrm{~km}$. Toulouse is a city located in South-West of France, subjected to both Oceanic and Mediterranean climatic influence. The average number of rainy days is 95.7 days/year and the average rainfall amount is $638.3 \mathrm{~mm} /$ year (1981-2010 Climate Normals). Salon de Provence is a city located in South-East of France, only subjected to Mediterranean climatic influences. The average number of rainy days is much lower than in Toulouse and is equal to 56.5 days/year (1981-2010 Climate Normals). The 


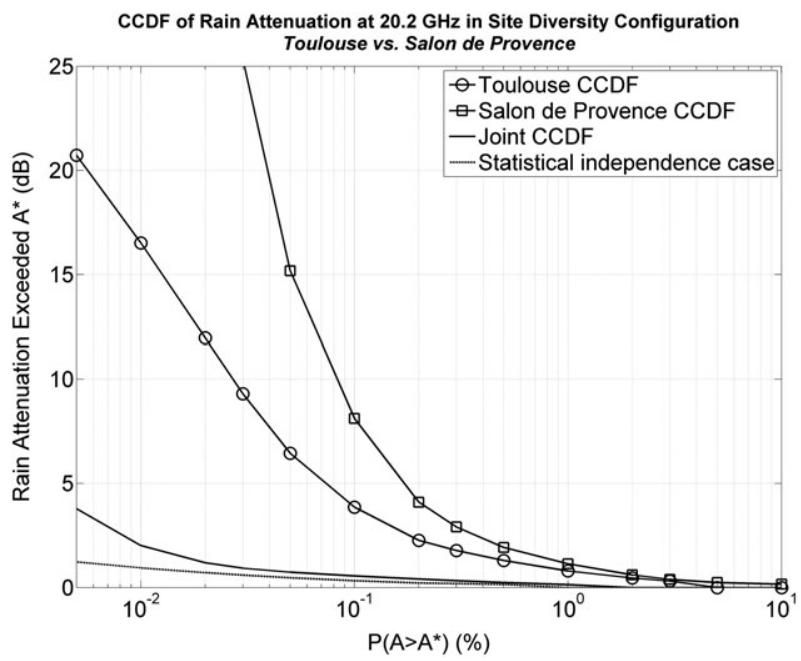

Figure 8. CCDF of rain attenuation at $20.2 \mathrm{GHz}$ in site diversity configuration between Toulouse and Salon de Provence.

average rainfall amount is comparable to the one in Toulouse and is equal to $579.9 \mathrm{~mm} / \mathrm{year}$ (19812010 Climate Normals). Salon de Provence is subjected to stronger summer storms which can dramatically increase rain attenuation on the radio link at percentages of time lower than $0.1 \%$.

Figure 9 shows the CCDF of rain attenuation at $20.2 \mathrm{GHz}$ in site diversity configuration between Toulouse and Aire sur l'Adour. The statistics have been computed from January 2014 to July 2014 over $95.04 \%$ of concurrent valid data. The correlation coefficient is equal to 0.0424 . Contrary to Figures 8 and 9 shows that concurrent single-site CCDF presents a similar shape. Indeed, Aire sur l'Adour is a city also located in the South-West of France and subjected to Atlantic Ocean influence. The Climate Normals are comparable to the one of Toulouse. Nevertheless, as for Figure 8, the spatial decorrelation effect is well noticeable with an experimental joint CCDF very close to the statistical independence case.

\subsection{Modelling of the spatial correlation of rain attenuation}

Starting from the experimental correlation coefficients between all sites, a modelling of the spatial correlation of long-term rain attenuation time series can be established. Two models have been analysed and fitted over the experimental data. The results are shown in Figure 10.

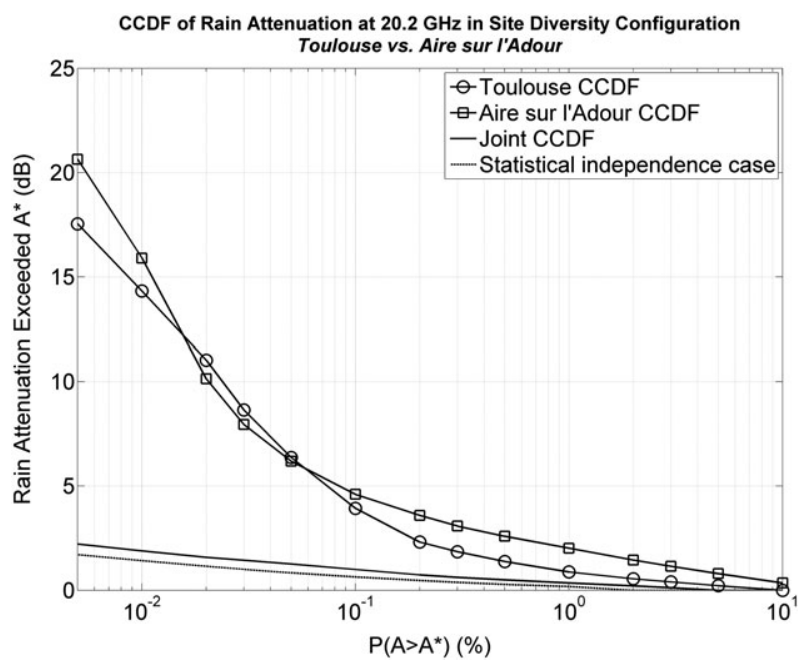

Figure 9. CCDF of rain attenuation at $20.2 \mathrm{GHz}$ in site diversity configuration between Toulouse and Aire sur l'Adour. 


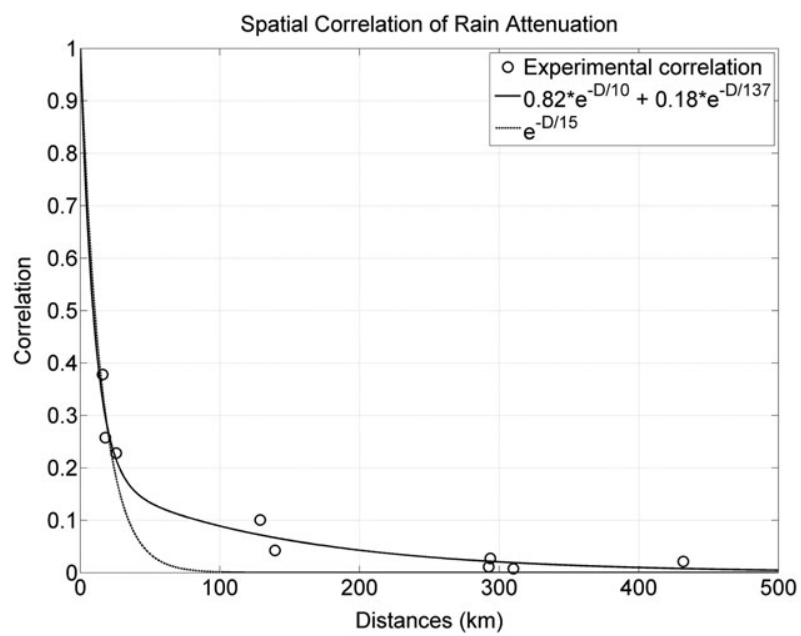

Figure 10. Modelling of the spatial correlation function of rain attenuation.

Table IV. RMS errors on Diversity Gains w.r.t Hodge/Paraboni-Barbaliscia models (results are marked by "_“ when the Hodge model is not applicable).

\begin{tabular}{|c|c|c|c|c|c|}
\hline $\begin{array}{l}\text { RMS errors on } \\
\text { Diversity Gains }\end{array}$ & Toulouse & Aussaguel & Le Fauga & Aire sur l'Adour & $\begin{array}{l}\text { Salon de } \\
\text { Provence }\end{array}$ \\
\hline Toulouse & & $0.33 / 0.19$ & $0.27 / 0.25$ & $-/ 0.21$ & $-/ 0.11$ \\
\hline Aussaguel & & & $0.25 / 0.24$ & $\begin{array}{l}\text { Not investigated due to } \\
\text { the lack of concurrent } \\
\text { measurements }\end{array}$ & $-/ 0.09$ \\
\hline Le Fauga & & & & $-/ 0.13$ & $-/ 0.11$ \\
\hline Aire sur l'Adour & & & & & $-/ 0.21$ \\
\hline Salon de Provence & & & & & \\
\hline
\end{tabular}

First of all, a simple decaying exponential function has been fitted showing that the model poorly reproduces the spatial correlation of rain attenuation at large scale. Then, a double decaying exponential function is analysed. The first part of the model reflects the behaviour of the correlation at small scale while the second part reflects the behaviour at large scale. Finally, the proposed model of the spatial correlation of unconditioned rain attenuation process $r_{A}$ is the following:

$$
r_{A}(D)=0.82 \exp \left(-\frac{D}{10}\right)+0.18 \exp \left(-\frac{D}{137}\right)
$$

$\mathrm{D}$ being the site separation distance in $\mathrm{km}$.

\section{COMPARISONS WITH ITU-R PREDICTION MODELS OF DIVERSITY GAIN}

The concept of diversity gain were introduced and characterized by Hodge in the 1970s [7], [28-30] in order to measure the advantage introduced by employing space diversity. It is defined as the difference in $\mathrm{dB}$ between the attenuation exceeded for a specified percentage of time on a single link and the attenuation simultaneously experienced on two or more links.

Two prediction models of site diversity performances are currently standardized into Rec. ITU-R P.618-12 [26]. The first one, given in Section 2.2.4.1 of [26], is based on the work of [31] and predicts the joint probability of rain attenuation for a set of two ground stations. It assumes lognormal distributions of rain intensity and of rain attenuation. In the following, it will be referred as the Paraboni-Barbaliscia model [31, 32]. The second one, given in Section 2.2.4.2 of [26], is an alternate simplified empirical method which directly predicts the diversity gain (in $\mathrm{dB}$ ) between pairs of sites with separation of less than $20 \mathrm{~km}$ [26]. It is based on the work of [30] and for which some regression coefficients have been refined. In the following, it will be referred as the Hodge model [30]. 


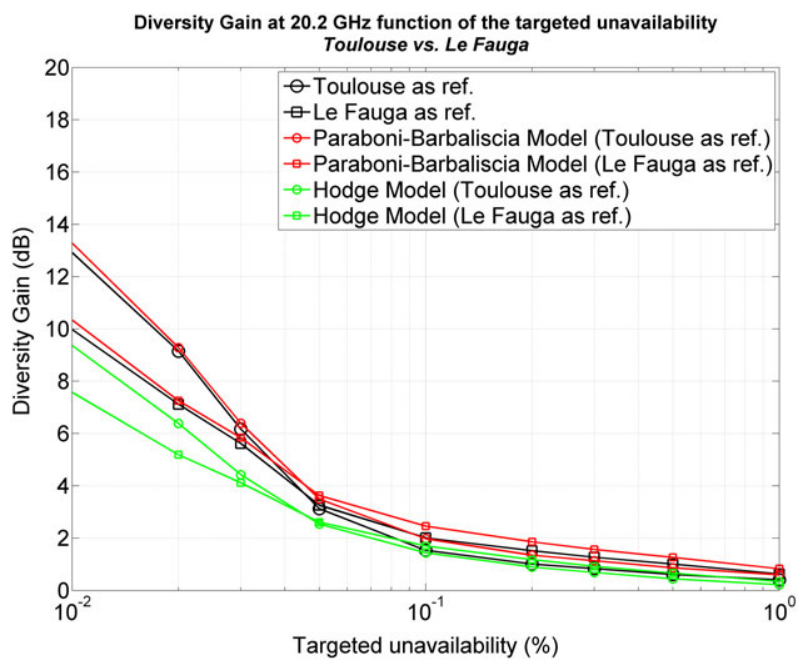

Figure 11. Comparison of experimental diversity gains at $20.2 \mathrm{GHz}$ w.r.t. ITU-R P.618-12 prediction models in Toulouse/Le Fauga configuration.

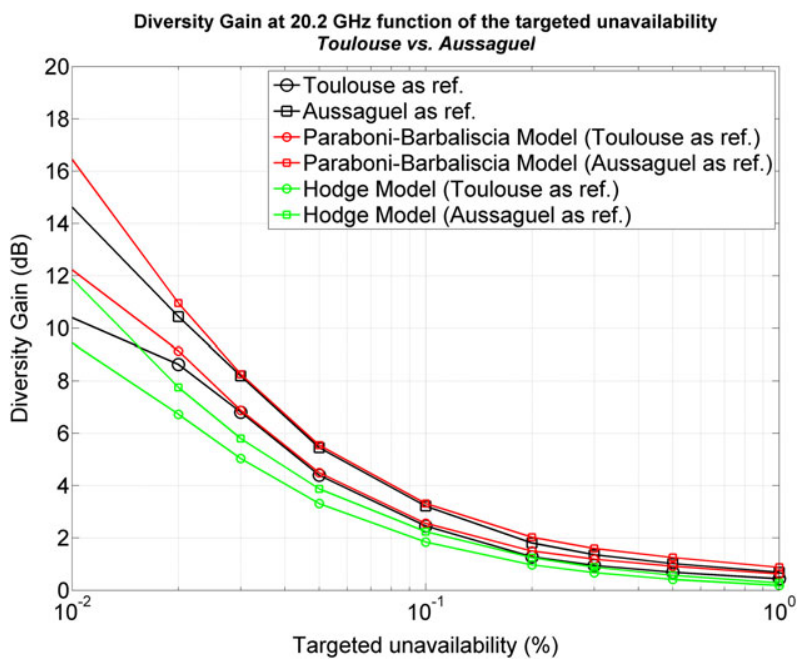

Figure 12. Comparison of experimental diversity gains at $20.2 \mathrm{GHz}$ w.r.t. ITU-R P.618-12 prediction models in Toulouse/Aussaguel configuration.

The RMS errors on Diversity Gains for both models (considering the relative error and probability levels in the range $0.01 \%-1 \%$ ) are summarized into Table IV.

\subsection{Small-scale site diversity configuration}

The two models have been tested w.r.t. the experimental diversity gain results. Note that the Hodge model is only shown for information in Toulouse/Le Fauga configuration as it is not strictly applicable (the site separation is slightly higher than $20 \mathrm{~km}$ ).

Figures 11-13 show the comparisons of the experimental diversity gains at $20.2 \mathrm{GHz}$ w.r.t. ITU-R P.618-12 prediction models respectively in Toulouse/Le Fauga, Toulouse/Aussaguel, and Le Fauga/Aussaguel configurations. Both beacon receivers are successively taken as reference in the computation of the diversity gain.

The Paraboni-Barbaliscia model generally presents a very good agreement with the experimental results with maximum absolute errors in the order of $1 \mathrm{~dB}$ in the range $0.01 \%-1 \%$. The Hodge model shows higher discrepancies. 


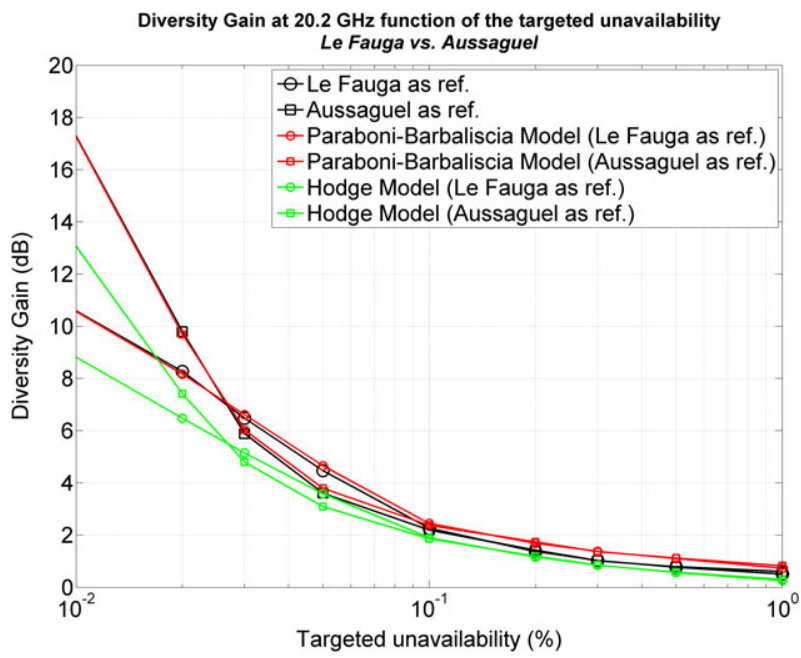

Figure 13. Comparison of experimental diversity gains at $20.2 \mathrm{GHz}$ w.r.t. ITU-R P.618-12 prediction models in Le Fauga/Aussaguel configuration.

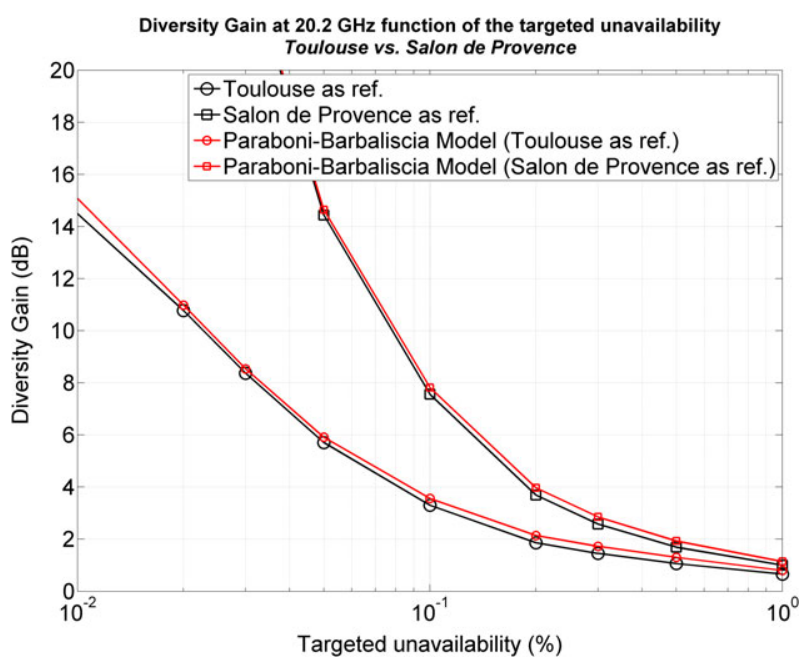

Figure 14. Comparison of experimental diversity gains at $20.2 \mathrm{GHz}$ w.r.t. the Paraboni-Barbaliscia prediction model in Toulouse/Salon de Provence configuration.

\subsection{Large-scale site diversity configuration}

As previously highlighted in Section 3.2., large-scale site diversity results are only illustrated in Toulouse/Salon de Provence and Toulouse/Aire sur l'Adour configurations. Only the ParaboniBarbaliscia model is tested due the limited scope of validity of the Hodge model.

Figures 14, 15 show the comparisons of the experimental diversity gains at $20.2 \mathrm{GHz}$ w.r.t. the Paraboni-Barbaliscia model respectively in Toulouse/Salon de Provence and in Toulouse/Aire sur l'Adour configurations. As for the results at small scale, the Paraboni-Barbaliscia model still shows a very good agreement with the experimental results in the range $0.01 \%-1 \%$.

\section{CONCLUSION}

From July 2013 to December 2014, ONERA and CNES conducted a 5 sites diversity experiment based on ASTRA 3B beacon signal. They have successively deployed, in the South of France, four more 20.2 GHz beacon receiver in addition to the one already operated in Toulouse since April 2011. Three 


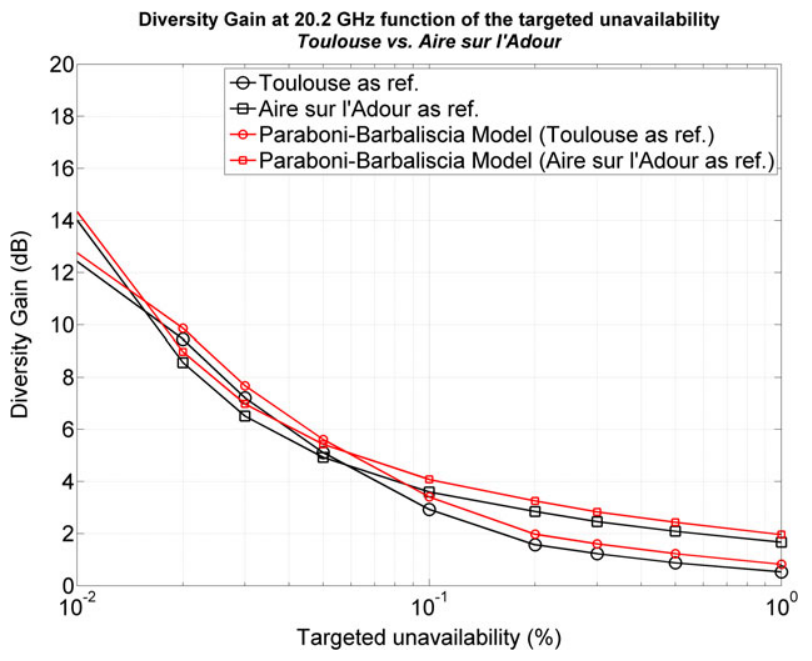

Figure 15. Comparison of experimental diversity gains at $20.2 \mathrm{GHz}$ w.r.t. the Paraboni-Barbaliscia prediction model in Toulouse/Aire sur l'Adour configuration.

of them, located in the area of Toulouse, allowed a small-scale site diversity experiment to be established. The last two beacon receivers were deployed in order to assess the tropospheric channel in large-scale site diversity configuration, and to characterize a different climatic area in Salon de Provence.

The results are given in terms of joint CCDF and diversity gain. They perfectly show the advantages introduced by employing site diversity mitigation technique. Moreover, two well-known standard prediction models of site diversity performances have been compared with the experimental results. The Paraboni-Barbaliscia model presents a very good agreement both at small-scale and large-scale.

At the end of 2014, three of the five beacon receivers were dismantled for other propagation studies in tropical and equatorial areas. However, since the beginning of 2015, ONERA has been operating two new beacon receivers able to receive the 19.7 and $39.4 \mathrm{GHz}$ Alphasat beacons. One of them is an outdoor dual-band dual-polarization beacon receiver (CNES) designed by the Joanneum Research Institute and deployed at Salon de Provence premises. The second one is an indoor $39.4 \mathrm{GHz}$ beacon receiver designed by ONERA and installed in Toulouse. At the end of the experiment, it should be possible to also characterize large-scale site diversity performances at $39.4 \mathrm{GHz}$.

\section{ACKNOWLEDGEMENTS}

This work has been carried out under the CNES R\&T study R-S11/TC-0005-023, "French Ka-band Site Diversity Experiment". The authors would like to thank Sébastien Rougerie and Franck Rousseau for their help when deploying CNES beacon receivers and radiometers. The authors are also very grateful to their colleagues from the Optical Department of ONERA, and more particularly to Thierry Huet, for providing the RPG HATPRO measurement data in Le Fauga.

\section{REFERENCES}

1. Berretta JG. KA-SAT, a reality. Proceedings 17th Ka Broadband Communications Navigation and Earth Observation Conference, Palermo, Italy, October 2011.

2. Freyer D. Ka-Band: Ready to make a business impact? Via Satell Mag 2008; XXIII(9):26-32.

3. Jeannin N et al. Smart gateways for Terabit/s satellite: Special issue on main results of Call-off Order 1 (CoO1) in SatNEx III. Int J Satell Commun Netw 2014; 32(2):93-106.

4. Kyrgiazos A, Evans B, Thompson P, Mathiopoulos T, Papaharalabos S. A terabit/second satellite system for European broad- band access: A feasibility study: Special issue on main results of Call-off Order 1 (CoO1) in SatNEx III. Int J Satell Commun Netw 2014; 32(2):63-92.

5. COST 255. Radiowave propagation modelling for new satcom services at Ku-band and above. ESA Publications Division, COST 255 Final Report, SP-1252, March 2002.

6. Hogg DC. Path diversity in propagation of millimeter waves through rain. IEEE Trans Ant Prop 1967; AP-15 (3):410-415. 
7. Hodge DB. Path diversity for reception of satellite signals. J Rech Atmos 1974; 8:443-449.

8. Harrold TW, Austin PM. The structure of precipitation systems-A review. J Rech Atmos 1974; 8(1-2):41-57.

9. Poiares Baptista JPV, Davies PG (Eds). OPEX Reference Book on Attenuation Measurement and Prediction. ESAWPP-083, Noordwijk: The Netherlands, 1994.

10. Giannone B, Saggese E, Matricciani E, Paraboni A. The ITALSAT propagation experiment. Space Commun Broadcast 1985; 3:221-231.

11. Paraboni A, Riva C, Valbonesi L, Mauri M. Eight years of ITALSAT copolar attenuation statistics at Spino d'Adda. Space Commun 2002 ISSN 0924-8625; 18:59-64.

12. Riva C. Seasonal and diurnal variations of total attenuation measured with the ITALSAT satellite at Spino d'Adda at 18.7, 39.6 and GHz. Int J Satell Commun Net 2004; 22:449-476. doi:10.1002/sat.784.

13. Ventouras S, Callaghan SA, Wrench CL. Long-term statistics of tropospheric attenuation from the Ka/U band ITALSAT satellite experiment in the United Kingdom. Radio Sci 2006; 41(2). doi:10.1029/2005RS003252.

14. Jorge F, Rocha A, Mota S. Evaluation of inter-annual variability of rainfall rate and rain attenuation based on the ITU Rec P.678. 9th European Conference on Antennas and Propagation (EuCAP), 2015; 1-4.

15. Garcia-Rubia JM, Riera JM, Garcia-del-Pino P, Siles GA, Benarroch A. Experimental assessment of slant-path rain attenuation variability in the Kaband. Int $J$ Satell Commun Net 2016; 34:155-170.

16. Amaya C, Nguyen T, Rocha A, Riera JM, Benarroch A, García del-Pino P, García-Rubia JM, Carrie G, Castanet L. Joint results of $20 \mathrm{GHz}$ recent earth-space propagation experiments in Canada and Europe. Proceedings of the 5th European Conference on Antennas and Propagation (EUCAP), 2011; 3384-3388.

17. Ishida T, Fugono N, Tabata J, Ohara M, Ishizawa T. Propagation experiment with Japanese satellite ETS-II (KIKU-2). Acta Astronaut 1980; 7(3):357-370.

18. Karasawa Y, Maekawa Y. Ka Band Earth Space Propagation Research in Japan. Proc IEEE 1997; 85(6):821-842.

19. Rogers DV, Crane RK. Review of propagation results from the advanced communications technology satellite (ACTS) and related studies. IEICE Trans Commun 2001; E84-B(9):2357-2368.

20. Crane RK, Wang X, Westenhaver DB, Vogel WJ. ACTS propagation experiment: experiment design, calibration and data preparation and archival, Special issue on Ka-band propagation effects on Earth-satellite links. Proc IEEE 1997; 85(6).

21. Crane RK. Propagation Handbook for Wireless Communication System Design. CRC Press LLC: Boca Raton, FL, USA, 2003.

22. Buti M, Fiebig UC. Joint statistics of slant-path attenuation in distant sites measured with ITALSAT at $40 \mathrm{GHz}$. Electron Lett 2006; 42(18):1014-1015.

23. Garcia-del-Pino P, Rocha A, Riera JM, Garcia-Rubia JM, Benarroch A. Joint Statistics of Rain Attenuation at $20 \mathrm{GHz}$ in Madrid and Aveiro. IEEE Antennas Wirel Propag Lett 2010; 9:799-802.

24. Goldhirsh G, Musiani BH, Dissanayake AW. Three-site space-diversity experiment at $20 \mathrm{GHz}$ using ACTS in the Eastern United States. Proc IEEE 1997; 85(6):970-980.

25. Callaghan S, Boyes B, Couchman A, Waight J, Walden CJ, Ventouras S. An investigation of site diversity and comparison with ITU-R recommendations. Radio Sci 2008; 43(4):1-8.

26. Propagation Data and Prediction Methods Required for the Design of Earth-Space Telecommunication Systems. International Telecommunication Union: Geneva, SwitzerlandITU-R P. 618-12, 2015.

27. Boulanger X, Gabard B, Casadebaig L, Castanet L. Four Years of Total Attenuation Statistics of Earth-Space Propagation Experiments at Ka-Band in Toulouse. IEEE Trans Ant Prop 2015; 63(5).

28. Hodge DB. Path diversity for earth-space communication link. Radio Sci 1978; 13(3):481-487.

29. Hodge DB. An empirical relationship for path diversity gain. IEEE Trans Ant Prop 1976; 24(2):250-251.

30. Hodge DB. An improved model for diversity gain on earth-space propagation paths. Radio Sci 1982; 17(6):1393-1399.

31. Paraboni A, Barbaliscia F. Multiple site attenuation prediction models based on the rainfall structures (meso- or synoptic scales) for advanced TLC or broadcasting systems. Proceedings XXVIIth URSI General Assembly, Maastricht, The Netherlands, 2002

32. Luglio M, Mancini R, Riva C, Paraboni A, Barbaliscia F. Large-scale site diversity for satellite communication networks. Int $J$ Satell Commun Netw 2002; 20(4):251-260. doi:10.1002/sat.723. 